\title{
Randomised controlled trial of doxorubicin-eluting beads vs conventional chemoembolisation for hepatocellular carcinoma
}

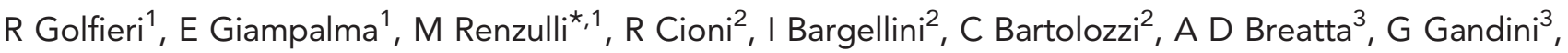 \\ R Nani ${ }^{4}$, D Gasparini ${ }^{5}$, A Cucchetti ${ }^{6}$, L Bolondi ${ }^{6}$ and $F$ Trevisani ${ }^{6}$ on behalf of the PRECISION ITALIA \\ STUDY GROUP ${ }^{7}$
}

${ }^{1}$ Radiology Unit, Department of Digestive Disease and Internal Medicine, S. Orsola-Malpighi Hospital, Bologna University, Albertoni Street 15, 40138 Bologna, Italy; ${ }^{2}$ Diagnostic and Interventional Radiology, Department of Oncology, Transplants and Advanced Technologies in Medicine, University of Pisa, Pisa, Italy; ${ }^{3}$ Diagnostic Imaging Division, Department of Medical and Surgical Disciplines University of Turin, AOU S. Giovanni Battista, Turin, Italy; ${ }^{4}$ CeLiveR, Ospedali Riuniti di Bergamo, Bergamo, Italy; ${ }^{5}$ Department of Radiologic Sciences, University Hospital, S. Maria della Misericordia, Udine, Italy and ${ }^{6}$ Department of Medical and Surgical Sciences, Alma Mater Studiorum - University of Bologna, Bologna, Italy

Background: Transcatheter arterial chemoembolisation (TACE) is the treatment of choice for intermediate stage hepatocellular carcinoma (HCC). Doxorubicin-loaded drug-eluting beads (DEB)-TACE is expected to improve the performance of conventional TACE (CTACE). The aim of this study was to compare DEB-TACE with cTACE in terms of time-to-tumour progression (TTP), adverse events (AEs), and 2-year survival.

Methods: Patients were randomised one-to-one to undergo cTACE or DEB-TACE and followed-up for at least 2 years or until death. Transcatheter arterial chemoembolisation was repeated 'on-demand'.

Results: We enrolled 177 patients: 89 underwent DEB-TACE and 88 cTACE. The median number of procedures was 2 in each arm, and the in-hospital stay was 3 and 4 days, respectively $(P=0.323)$. No differences were found in local and overall tumour response. The median TTP was 9 months in both arms. The AE incidence and severity did not differ between the arms, except for postprocedural pain, more frequent and severe after CTACE $(P<0.001)$. The 1 - and 2 -year survival rates were $86.2 \%$ and $56.8 \%$ after DEB-TACE and $83.5 \%$ and $55.4 \%$ after CTACE $(P=0.949)$. Eastern Cooperative Oncology Group (ECOG), serum albumin, and tumour number independently predicted survival $(P<0.05)$.

Conclusions: The DEB-TACE and the cTACE are equally effective and safe, with the only advantage of DEB-TACE being less postprocedural abdominal pain.

Worldwide, hepatocellular carcinoma (HCC) is the fifth most common cancer in men and the sixth in women, representing the third leading cause of tumour-related death (El-Serag, 2012).
The majority of HCCs are associated with liver cirrhosis, and the prognosis is so poor that mortality and incidence are fairly similar (El-Serag, 2012).

\footnotetext{
*Correspondence: Dr M Renzulli; E-mail: matteo.renzulli@aosp.bo.it

${ }^{7}$ Members of the PRECISION ITALIA STUDY GROUP are listed before References.
}

Received 20 December 2013; revised 9 March 2014; accepted 20 March 2014; published online 17 June 2014

(C) 2014 Cancer Research UK. All rights reserved 0007 - 0920/14 
According to Barcelona Clinic Liver Cancer (BCLC) tumour staging and management (Bruix and Llovet, 2002), endorsed by the American (AASLD) and European (EASL-EORTC) guidelines for HCC management (Bruix and Sherman, 2005; Llovet et al, 2012), transcatheter arterial chemoembolisation (TACE) is recommended as first-line therapy for unresectable intermediate-stage HCC (stage B). This evidence-based position reflects the results of three studies showing that TACE significantly improves patient survival as compared with best supportive care (Llovet et al, 2002; Lo et al, 2002; Llovet and Bruix, 2003). Despite the widespread use of screening programmes, $60-70 \%$ of HCCs are detected when curative treatments (surgery or ablation) are precluded by the cancer burden or contraindications (Bruix and Llovet, 2002; Takayasu et al, 2012). In fact, TACE is the most common treatment modality used in HCC patients, and almost half of TACEs are carried out for early tumours unsuitable for curative treatment (Varela et al, 2010).

Agreement regarding the best TACE technique has not yet been reached, and various options concerning patient selection, delivery systems, selectivity of treatment, drugs and embolic agents injected, and repetition schedules (predefined $v s$ 'on demand') are still in use (Marelli et al, 2007), making the results reported in the literature very inhomogeneous.

Transcatheter arterial chemoembolisation with calibrated doxorubicin-carrying microspheres, DC-Beads (DEB-TACE), have been introduced as a novel device capable of ensuring more sustained and tumour-selective drug delivery and permanent embolisation (Varela et al, 2007), which could satisfy the requirement for standardising treatment regimens. A multicentre international randomised trial (Lammer et al, 2010) comparing the short-term outcomes of DEB-TACE and conventional TACE (cTACE) in terms of toxicity and radiologic tumour response indicated some advantages of the first technique, particularly in 'fragile' subgroups, such as Child-Pugh B and performance status (PS) $>0$ patients, and in those with bilobar or recurrent tumours. Despite these appealing premises, the superiority of DEB-TACE over CTACE in terms of patient survival has yet to be demonstrated. Two retrospective studies (Dhanasekaran et al, 2010; Song et al, 2012), affected by several biases, have suggested the superiority of DEB-TACE whereas a small randomised controlled trial has not confirmed this superiority (Sacco et al, 2011).

To elucidate this key point, we conducted a prospective, multicentre randomised controlled study involving a large series of cirrhotic patients with HCC not amenable to curative therapy. The aim was to compare the 2-year survival achievable with DEBTACE and cTACE in homogeneous patients.

\section{MATERIALS AND METHODS}

Study population. Patients $>18$ years of age, with HCC unsuitable for curative treatment or had failed/recurred after resection/ablation and diagnosed by biopsy or according to the AASLD criteria (Bruix and Sherman, 2005), were eligible for the study. Causes of non-qualification for curative treatments were contraindications to liver transplant; poor liver function (ChildPugh class B or MELD score $>10$ ), severe comorbidities and patient refusal for resection; critical location or non-visibility at ultrasonography of nodules and not permissive clotting/platelet count for ablation. The inclusion criteria were patients belonging to class Child-Pugh A or B (score 7) and with an Eastern Cooperative Oncology Group (ECOG) PS $\leqslant 1$, no previous treatment on target lesions (prior treatments on non-target lesions were accepted). The exclusion criteria were infiltrative HCC, portal vein thrombosis, ascites, F3 oesophageal varices, advanced liver disease (bilirubin levels $\geqslant 2.5 \mathrm{mg} \mathrm{dl}^{-1}$, albumin $\leqslant 30 \mathrm{gl}^{-1}$, platelets $\leqslant 50 \times 10^{9}$ per litre, INR $>1.5$ ), other tumours in the previous 5 years, and contraindications to arteriography or TACE. Previous locoregional treatment (including resection) of non-target lesions did not preclude enrolment.

All patients provided written informed consent. This study was conducted in accordance with the Good Clinical Practice principles and the Declaration of Helsinki. Independent Data and Safety Monitoring Committees were established to monitor efficacy and safety data. Ethics committee approval was obtained. The study was registered at www.clinicaltrials.gov (NCT00936689).

Study design. The study, called 'Precision Italia', was a multicentre, prospective, randomised, open-label, active-controlled study in which patients were randomised one-to-one to undergo cTACE or DEB-TACE. The study required a post-TACE follow-up for at least 2 years or until death. Upon enrolment, alphanumeric identification codes were assigned to the patients who were then randomised to one of the two treatments. The randomisation was stratified according to Child-Pugh class and BCLC stage and it was centralised at the Investigational Drug Service of the Pharmacy of the Bologna center, allocating the first and lowest randomisation code available, and generating the randomisation list.

Patients underwent TACE as follows: (1) patients of the cTACE group (Arm A) received a mixture of dry epirubicin (Farmorubicin; Pfizer, Latina, Italy) manually emulsified with iodised oil (Lipiodol; Guerbet, Milan, Italy) in a proportion of 1 to 1 vial (50 mg of drug with $10 \mathrm{ml}$ of Lipiodol) to a maximum administered dose of $75 \mathrm{mg}$, followed by embolisation with absorbable gelatin sponge particles (Gelita-Spon; Gelita Medical, Amsterdam, The Netherlands); (2) patients of the DEB-TACE group (Arm B) received DC-Beads (Biocompatibles, Farnham, Surrey, UK), $100-300 \mu \mathrm{m}$ in diameter (each vial was loaded with $50 \mathrm{mg}$ of a doxorubicin solution; Pfizer) mixed with non-ionic contrast medium. In both arms, chemoembolisation was performed as selectively as possible (segmental (selective) or subsegmental (superselective)) using a 2.7-2.8 French microcatheter.

In the cTACE group, the mixture was injected until saturation of the tumour feeding arteries, and the subsequent embolisation end point was to reduce the residual blood flow. In the DEB-TACE group, we injected the loaded microspheres until full saturation of the tumour feeding arteries, without injection of other embolic agents.

Transcatheter arterial chemoembolisation was repeated 'on demand' upon demonstration of a persistent viable tumour (i.e., the absence of complete response (CR)) or intra-hepatic distal recurrence at imaging follow-up, provided that liver function had not deteriorated (Child-Pugh score $>7$ ).

Efficacy evaluation. The response was evaluated by Multidetector Computer Tomography (MDCT) or Magnetic Resonance Imaging (MRI) as local (per-lesion) tumour response and overall (perpatient) response, according to the EASL criteria (until 2010) and, subsequently, to the modified RECIST (mRECIST) (Lencioni and Llovet, 2010), at 1 month after each TACE and, thereafter, every 3 months, for at least 2 years. An objective response (OR) was defined as CR plus partial response (PR), while disease control (DC) was defined as OR plus stable disease (SD).

Study aims and hypothesis. The primary aim of the study was to compare 2-year survival between the two study arms. The secondary end points were radiological tumour response, timeto-tumour progression (TTP: interval between randomisation and radiological tumour progression), impact on PS and liver function, number of treatments, duration of in-hospital stay and need for other types of treatment of residual/recurrent tumours. For obvious ethical reasons and since our investigation aimed at 
achieving results similar to those achievable with the HCC therapies usually adopted in our country (pragmatic trial), the study design allowed referral physicians to treat tumour persistence/progression after at least two TACEs with alternative treatments (ablation, surgery or sorafenib administration). According to the rules of the Italian Drug Regulatory Agency (AIFA), sorafenib was dispensed to patients who were in Child-Pugh class $\mathrm{A}$ at the time of tumour progression.

Safety evaluation. Adverse events (AEs) and serious adverse events (SAEs) were monitored and recorded. Adverse events were assessed during and after each treatment, and at all follow-up visits and graded according to the NCI Common Terminology Criteria for Adverse Events (CTCAE) version 3.0. The AEs occurring within 4 weeks after TACE were considered as treatment related; whenever they occurred after 4 weeks, they were reported only if a causal correlation was suspected.

Statistical analysis. The study was designed to demonstrate the superiority of DEB-TACE over CTACE in 2-year survival, assuming a $40 \%$ survival rate in the cTACE arm and a $20 \%$ improvement in the DEB-TACE arm. To detect this difference, with $80 \%$ power and a $5 \%$ significance level, we calculated that 214 patients, 107 per treatment arm, had to be enrolled (Fleiss, 1973).

Two formal interim analyses, planned after $\sim 50 \%$ and $75 \%$ of enrolment, were conducted by an external statistician to assess survival at 24 months post treatment. The first interim analysis, involving 116 patients (57 cTACE and 59 DEB-TACE cases; 54\% of the planned sample size), showed no difference between the two arms (Mantel-Haenszel, $P=0.853$ ). Similarly, the second interim analysis, involving 177 patients (88 cTACE and 89 DEB-TACE), showed no difference between arms (Mantel-Haenszel, $P=0.949$ ). A reviewer committee, formed by two experts external to the study, and an external statistician, was thus consulted on the utility of continuing enrolment. Since it was highly unlikely that extended enrolment would reveal the superiority of DEB-TACE, the committee recommended stopping the trial for futility (Pocock, 1992). The results presented here are therefore derived from a population of 177 patients, representing $83 \%$ of the original planned sample size (Figure 1).

Survival and safety analyses included all randomised patients who underwent at least one TACE (intention-to-treat analysis). The Kaplan-Meier method was used to calculate survival curves, and the Mantel-Haenszel test was used for treatment comparisons. For patients who became transplantable after a downstaging programme, survival was censored at the day before surgery.

Categorical variables are reported in numbers and percentages. The variable distribution was checked with the KolmogorovSmirnov test, and the values are reported as mean \pm standard deviation, or median and range, as appropriate. Comparisons between groups were carried out using the Fisher's exact test, ANOVA, or Mann-Whitney. In addition to $P$-values, which are affected by sample size, effect sizes (or standardised differences) were computed: values $<0.1$ indicated very small differences, $0.1-$ 0.3 small differences, $0.3-0.5$ moderate differences, and $>0.5$ large differences (Burnand et al, 1990).

Univariate and multivariate Cox proportional hazard analyses were used to assess the prognostic role of baseline patient characteristics. Variables associated $(P<0.10)$ with overall survival at univariate analysis were entered into the multivariate regression model. The regression model was further explored by including the 'primary exposure variable' (TACE treatment) even if it had a $P$-value of $>0.10$ at univariate analysis. A two-tailed $P$-value of $<0.05$ was considered as statistically significant. Statistical evaluations were carried out using the SPSS version 13.0 for Windows (IBM, Armonk, NY, USA).

\section{RESULTS}

Patients were enrolled between March 2008 and December 2010; the date of the last follow-up evaluation was December 2012. The flow chart of the study is summarised in Figure 1. Fifty-eight $(32.7 \%)$ randomised patients had undergone previous treatments, 31 in the cTACE group (40 treatments in all: 15 cTACE, 16 percutaneous treatments, and 9 resections) and 27 in the DEBTACE group (37 treatments in all: 9 cTACE, 21 percutaneous treatments, and 7 resections $)(P=0.524)$. In all, $36(20 \%)$ patients received subsequent treatments that were equally distributed in the two arms $(P=0.404)$ : liver transplant in 6 cTACE and 4 DEBTACE patients, sorafenib in 14 cTACE and 9 DEB-TACE cases, ablative procedures in $1 \mathrm{cTACE}$ and 1 DEB-TACE patient, and resection in 1 DEB-TACE subject.

During the 2-year follow-up after TACE, 73 (41.2\%) patients died, $36(40.9 \%)$ in the cTACE arm, and $37(41.6 \%)$ in the

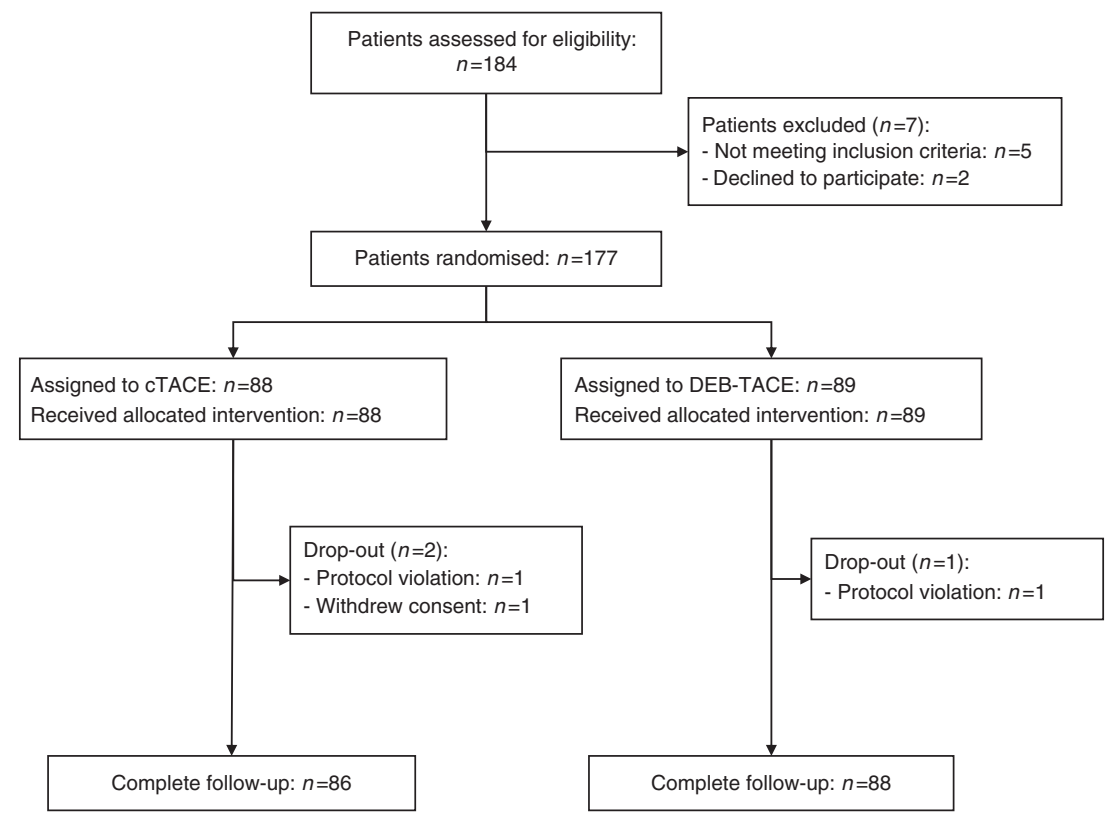

Figure 1. Summary flow chart of the study. 
DEB-TACE arm. The causes of death were sepsis in 1 patient (1.4\%), tumour progression in $49(67.1 \%)$, liver failure in 17 $(23.3 \%)$, and other causes in $6(8.2 \%)$.

Baseline patient characteristics are reported in Table 1 . No significant differences were observed in clinical and tumoural characteristics between the two groups. All the 46 patients classified as BCLC C were allocated to this stage due to ECOG-1.

Patients in the cTACE arm received $47.2 \pm 14.6 \mathrm{mg}$ of epirubicin and $10.3 \pm 3.8 \mathrm{ml}$ of Lipiodol whereas patients in the DEB-TACE arm received $57.8 \pm 24.1 \mathrm{mg}$ of doxorubicin. Two-thirds of the patients in each arm underwent segmental TACE treatment.

After the 30-day follow-up visit, 21 (24.1\%) cTACE and 28 (31.5\%) DEB-TACE patients repeated the procedure $(P=0.315)$. At the 3-month assessment, 15 (18.8\%) cTACE and $13(17.3 \%)$ DEB-TACE patients were re-treated $(P=0.838)$. A similar re-treatment rate between the groups was maintained also at the 6-month $(P=0.376)$ and the 9-month assessments $(P>0.999)$. As a result, the median number of treatments was 2 in both the cTACE (range: 1-4) and the DEB-TACE arms (range: 1-5).

Tumour response. At 30 days, radiological evaluation of the tumour response was available in all but one patient who had died after the first cTACE from septic shock. A local CR (of the target lesions) was observed in 101 patients $(57.1 \%)$, overall CR in 91 (51.4\%), PR in 66 (37.3\%), SD in $6(3.4 \%)$, and progressive disease (PD) in 13 (7.3\%). Thus, OR was obtained in 157 patients $(88.7 \%)$ and DC in 163 (92.1\%). No significant differences were found in the rates of local (CR, OR, and DC) (Figure 2) and overall tumour responses during the follow-up period $(P>0.05$ in all cases), except for a more frequent overall $\mathrm{CR}$ at 1 month after cTACE than after DEB-TACE (59.8\% vs 43.8\%; $P=0.036$ ) (Figure 3). Conversely, a trend towards a higher rate of local CR after DEB-TACE was observed at 6 months $(68.7 \%$ vs $63.5 \%)$. This trend, however, conflicted with a tendency to a higher rate of overall CR after cTACE at the same time point (58.1\% vs 52.2\%), due to the lower occurrence of new nodules in cTACE (5 out of 20,25\%) than in DEB-TACE patients ( 9 out of $23,39 \%$ ) at the time of progression, although this difference did not reach statistical significance $(P=0.353)$.

We also conducted post hoc analyses on overall tumour response in some patient subgroups (Table 2). At 30 days, an overall CR was significantly more frequent after CTACE than after DEB-TACE in ECOG-0 $(P=0.014)$ and BCLC class A $(P=0.027)$ patients.

TTP and survival. For all patients, the median TTP was 9 months (95\% CI: 7.3-10.7), namely 9 months (95\% CI: 6.3-11.7) in the cTACE arm and 9 months (95\% CI: 6.8-11.2) in the DEB-TACE $\operatorname{arm}(P=0.766)$.

In the entire population, the 1 - and 2 -year survival rates were $84.8 \%$ and $56.2 \%$, respectively. The corresponding figures were $83.5 \%$ and $55.4 \%$, respectively after cTACE, and $86.2 \%$ and $56.8 \%$ after DEB-TACE $(P=0.949)$ (Figure 4$)$. Based on these figures, the median survival projection was 29 months for all patients, 28 months for cTACE patients and 29 months for DEB-TACE patients. A post hoc analysis on survival showed that the 1- and 2 -year survival rates were $88.6 \%$ and $68.6 \%$ in BCLC A, $85.4 \%$ and $64.2 \%$ in BCLC B, and $77.5 \%$ and $50.1 \%$ in BLCL C patients ( $P$ for trend $=0.033)$. Conversely, the 1 - and 2 -year survival rates were not significantly affected by the 'naïve' status, being $89.5 \%$ and $65.9 \%$ in naïve cases against $75.4 \%$ and $55.6 \%$ in previously treated individuals $(P=0.156)$.

Table 1. Baseline clinical and tumour characteristics of the study population randomised to receive cTACE or DEB-TACE

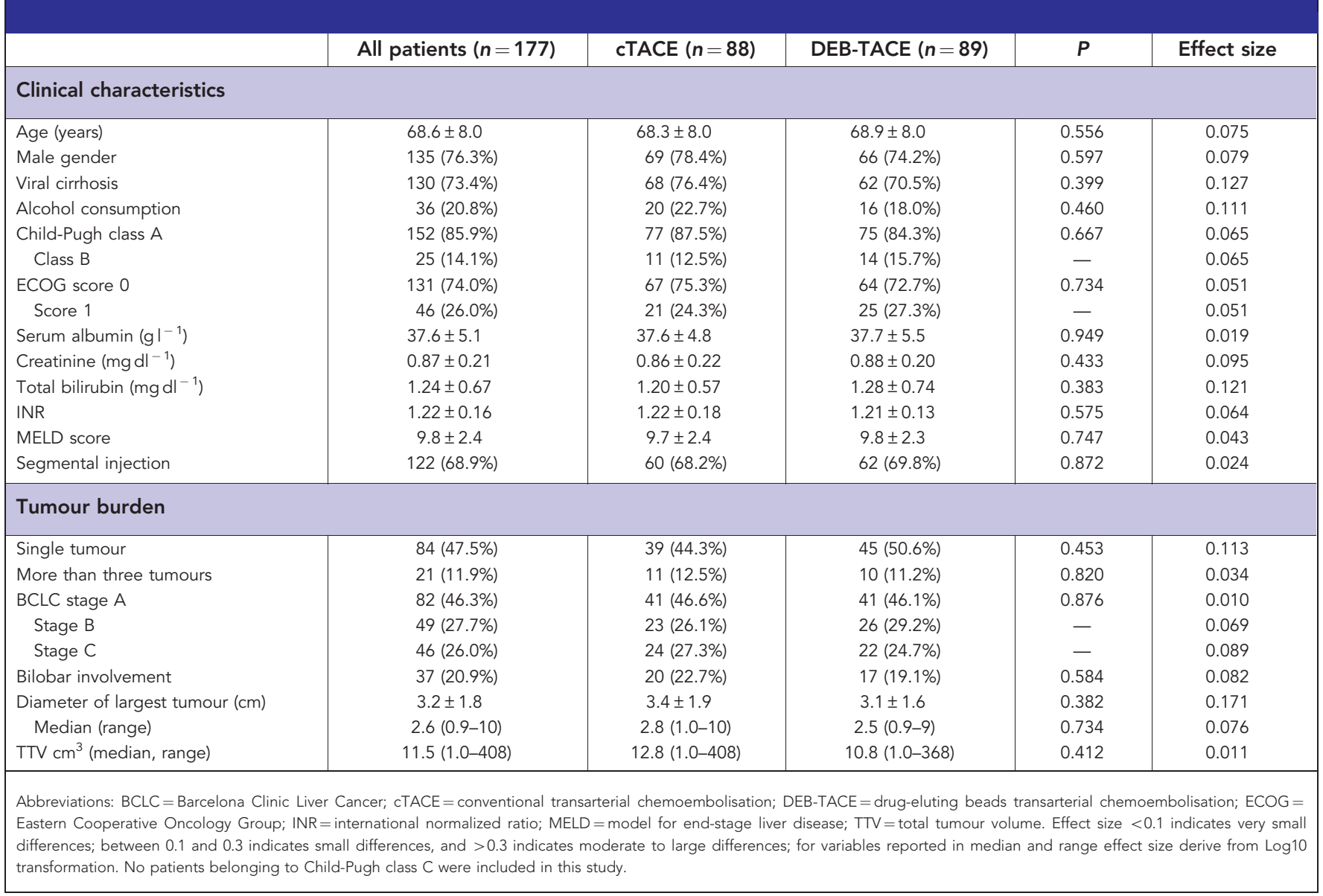



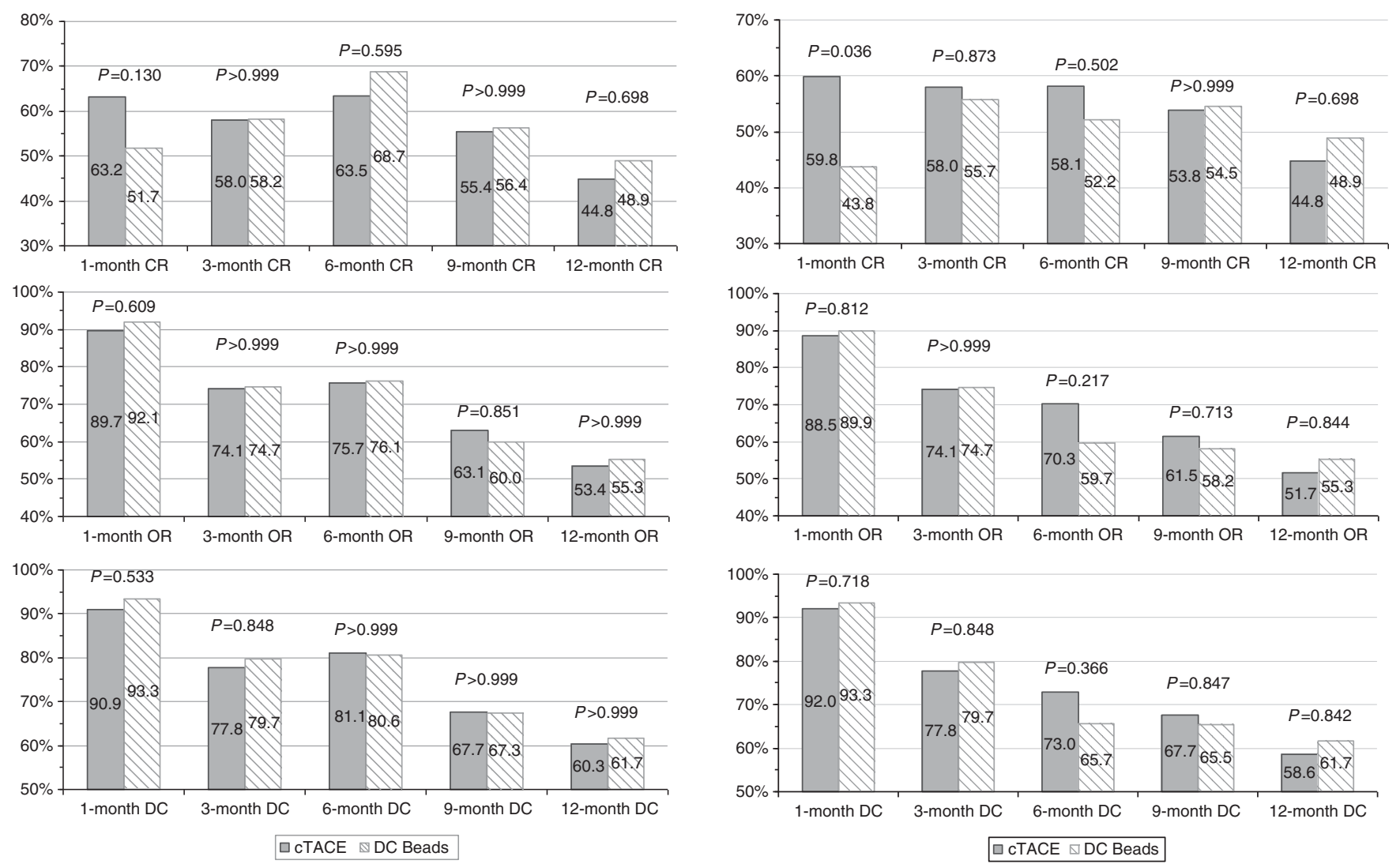

Figure 2. Local response of lesions treated. Abbreviations: $\mathrm{CR}=$ complete response; $\mathrm{DC}=$ disease control; $\mathrm{OR}=$ objective response.

The type of TACE did not affect patient survival at the univariate regression analysis whereas ECOG-1, low serum albumin, and multiple tumours independently and adversely conditioned survival (Table 3). When the multivariate model was forced to include the TACE type, ECOG-1, serum albumin, and multiple tumours were confirmed as independent prognostic factors, and the treatment type remained insignificant $(P=0.410$; Hazard ratio: 0.821; 95\% CI: 0.513-1.313) (Table 3).

Safety. Adverse events are reported in Table 4. There was no significant difference in the incidence of all AEs except postprocedural pain, which was two-fold more frequent and more severe in the cTACE arm than in the counterpart. The SAEs were rare in both arms $(<7 \%)$. A significant worsening of liver function at 1 month was exceptional (3.4\%) and equally distributed between the two arms.

In-hospital stay length. The median in-hospital stay was 4 days (range: 1-26) for cTACE patients and 3 days (range: 1-34) for DEB-TACE patients $(P=0.323)$.

\section{DISCUSSION}

Transcatheter arterial chemoembolisation is considered as the standard treatment for patients with HCC in the BCLC intermediate stage (Bruix and Llovet, 2002; Llovet et al, 2012) but it is also used in early-stage HCC patients excluded from potentially curative treatment. In fact, as in other studies (Varela et al, 2010; Burrel et al, 2012; Takayasu et al, 2012), early HCC patients represented a notable proportion of our cases. Conventional TACE suffers from several technical problems, such as lack of standardisation of the procedure and escape of the

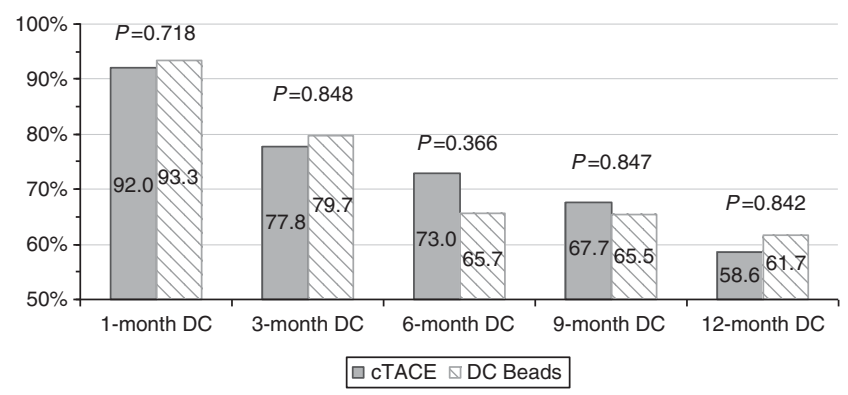

Figure 3. Overall response of the entire liver according to $\mathrm{mRECIST}$ criteria. Abbreviations: $\mathrm{CR}=$ complete response; $\mathrm{DC}=$ disease control; $\mathrm{OR}=$ objective response.

chemotherapeutic agent from the treated portion of liver. These limitations are theoretically reduced by DEB-TACE. The current study is a head-to-head comparison of survival between DEBTACE and well-standardised cTACE which, until now, has only been addressed by a small randomised study (Sacco et al, 2011).

Our study did not demonstrate any difference in local and overall response and DC between CTACE and DEB-TACE, except at 1 month when cTACE achieved a higher rate of overall CR. As this superiority disappeared thereafter, when lipiodol staining decreased, it probably represents a spurious benefit due to the 'masking effect' of lipiodol at the first post-TACE evaluation with MDCT. This potential inaccuracy in the initial tumour response was accepted at the time we designed the study for two reasons; first, in clinical practice MDCT is more easily available than MR, and is one of tools recommended to control TACE results (Bruix and Sherman, 2005); second, the primary objective of the study was to compare the survival of patient undergoing the two procedures.

The 3-month OR rate (about 74\%), seen in both treatment arms, is in line with what was reported in our previous study (Golfieri et al, 2013) using cTACE, and by Song et al (2012) with DEBTACE. In the Korean series, the OR achieved with cTACE was much lower (49.4\%) than ours, possibly due to a defectively standardised procedure. These authors also reported a better response with DEB-TACE in intermediate-stage but not in earlystage tumours, suggesting that this technique could facilitate treatment of larger lesions. Such a stage-dependent difference in the efficacy between the two types of TACE was not confirmed by our study, which did not detect any differences in the CR rates between treatment arms according to BCLC stage, lobar/bilobar tumoural extension or Child-Pugh class.

Our results also conflict with the first multicentric randomised European trial (Lammer et al, 2010), reporting a better response at 


\begin{tabular}{|c|c|c|c|c|}
\hline & $\begin{array}{l}\text { cTACE } \\
\text { number/number } \\
\text { at risk }(\%)^{a}\end{array}$ & $\begin{array}{l}\text { DEB-TACE } \\
\text { number/number } \\
\text { at risk }(\%)\end{array}$ & $P$ & $\begin{array}{l}\text { Effect } \\
\text { size }\end{array}$ \\
\hline \multicolumn{5}{|c|}{ Child-Pugh class } \\
\hline \multicolumn{5}{|l|}{ A } \\
\hline 1 month & $45 / 77$ (58.4\%) & $34 / 75(45.3 \%)$ & 0.144 & 0.291 \\
\hline 3 months & $41 / 72$ (56.9\%) & $38 / 67$ (56.7\%) & $>0.999$ & 0.005 \\
\hline 6 months & $39 / 67(58.2 \%)$ & $29 / 59(49.2 \%)$ & 0.371 & 0.201 \\
\hline \multicolumn{5}{|l|}{ B7-B8 } \\
\hline 1 month & 7/10 (70.0\%) & $5 / 14(35.7 \%)$ & 0.214 & 0.791 \\
\hline 3 months & $6 / 9(66.7 \%)$ & 6/12 (50.0\%) & 0.660 & 0.382 \\
\hline 6 months & 4/7 (57.1\%) & $6 / 8(75.0 \%)$ & 0.608 & 0.447 \\
\hline \multicolumn{5}{|c|}{ Bilobar involvement } \\
\hline \multicolumn{5}{|l|}{ Absent } \\
\hline 1 month & $45 / 67(67.2 \%)$ & $36 / 72(50.0 \%)$ & 0.058 & 0.395 \\
\hline 3 months & 40/64 (62.5\%) & $37 / 63(58.7 \%)$ & 0.718 & 0.087 \\
\hline 6 months & $38 / 62$ (61.3\%) & 28/52 (53.8\%) & 0.451 & 0.168 \\
\hline \multicolumn{5}{|l|}{ Present } \\
\hline 1 month & $7 / 20$ (35.0\%) & $3 / 17$ (17.6\%) & 0.288 & 0.508 \\
\hline 3 months & 7/17 (41.2\%) & $7 / 16$ (43.8\%) & $>0.999$ & 0.058 \\
\hline 6 months & 5/12 (41.7\%) & 7/15 (46.7\%) & $>0.999$ & 0.112 \\
\hline \multicolumn{5}{|l|}{ ECOG } \\
\hline \multicolumn{5}{|l|}{0} \\
\hline 1 month & $38 / 63(60.3 \%)$ & $25 / 67(37.3 \%)$ & 0.014 & 0.517 \\
\hline 3 months & $37 / 60(61.7 \%)$ & $29 / 58$ (50.0\%) & 0.266 & 0.262 \\
\hline 6 months & $31 / 57(54.4 \%)$ & $24 / 50(48.0 \%)$ & 0.564 & 0.141 \\
\hline \multicolumn{5}{|l|}{1} \\
\hline 1 month & $14 / 24(58.3 \%)$ & $14 / 22(63.6 \%)$ & 0.769 & 0.123 \\
\hline 3 months & $10 / 21(47.6 \%)$ & 6/15 (28.6\%) & 0.208 & 0.558 \\
\hline 6 months & $12 / 17$ (29.4\%) & $11 / 17$ (64.7\%) & $>0.999$ & 0.148 \\
\hline \multicolumn{5}{|l|}{ BCLC } \\
\hline \multicolumn{5}{|l|}{ Stage A } \\
\hline 1 month & $32 / 40$ (80.0\%) & $20 / 41$ (48.8\%) & 0.005 & 0.689 \\
\hline 3 months & $26 / 40(65.0 \%)$ & $21 / 36$ (58.3\%) & 0.639 & 0.137 \\
\hline 6 months & $27 / 39$ (69.2\%) & 16/31 (51.6\%) & 0.147 & 0.366 \\
\hline \multicolumn{5}{|l|}{ Stage B } \\
\hline 1 month & $6 / 23(26.1 \%)$ & $5 / 26(19.2 \%)$ & 0.734 & 0.164 \\
\hline 3 months & $11 / 20(55.0 \%)$ & 8/22 (36.4\%) & 0.352 & 0.380 \\
\hline 6 months & 4/18 (22.2\%) & 8/19 (42.1\%) & 0.295 & 0.436 \\
\hline \multicolumn{5}{|l|}{ Stage C } \\
\hline 1 month & $14 / 24$ (58.3\%) & $14 / 22(63.6 \%)$ & 0.769 & 0.109 \\
\hline 3 months & 10/21 (47.6\%) & 15/21 (71.4\%) & 0.208 & 0.500 \\
\hline 6 months & $12 / 17(70.6 \%)$ & $11 / 17$ (64.7\%) & $>0.999$ & 0.126 \\
\hline \multicolumn{5}{|c|}{ 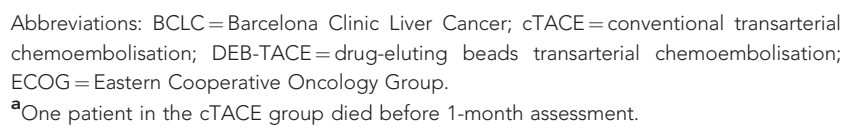 } \\
\hline
\end{tabular}

6 months with DEB-TACE in more compromised patients (ChildPugh B, ECOG-1, bilobar, or recurrent disease). However, in this trial the SAE incidence was $\sim 20 \%$ in both cTACE and DEB-TACE patients, leading to treatment discontinuation in $13 \%$ of cases. This trial also reported significantly reduced side effects after DEBTACE. These figures are much higher than ours (6.2\%), regardless of the type of TACE we employed. Such different findings could be related both to the TACE reiteration at 2-month intervals

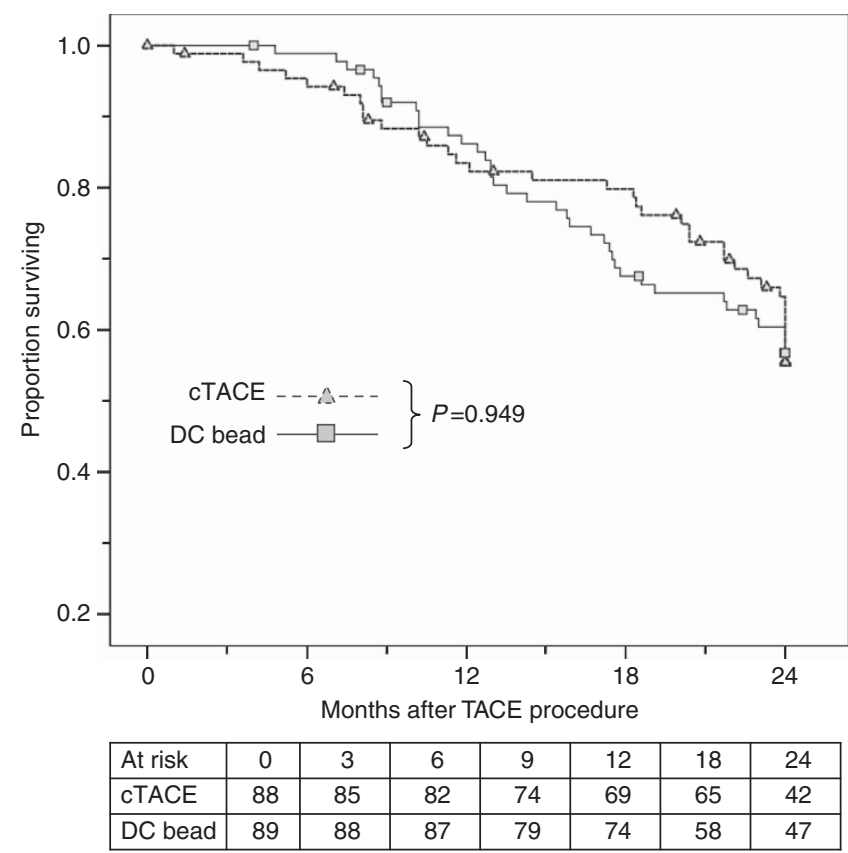

Figure 4. Patient survival after CTACE (triangle plot) and DEB-TACE (square plot).

regardless of tumour response (82\% of patients received a second TACE and $\sim 60 \%$ a third TACE), and to the high amount of Doxorubicin (up to $150 \mathrm{mg}$ per course) utilised in the European study. In this respect, it is important to note that we injected the microspheres (as well as the mixture of epirubicin and Lipiodol) through microcatheters (2.7-2.8 French) and until full saturation of the tumour feeding arteries was obtained. This allowed us reaching the maximal administrable dose in a superselective modality using the smallest microspheres (100-300 $\mu \mathrm{m})$, and hence avoiding the risk of under-treating target lesions, as indirectly confirmed by the high rate of $\mathrm{CR}$ we obtained. Therefore, the higher drug dose utilised in the European trial was probably due to a less selective approach and/or a greater median size of the lesions allowing the injection of larger spheres $(300-700 \mu \mathrm{m})$. Finally, a recent meta-analysis based on seven studies (693 patients) demonstrated that the two procedures had equivalent results, strongly supporting our findings regarding the lack of difference in tumour response between the two procedures (Gao et al, 2013).

As expected, in our study, moderate and severe periprocedural pain was less frequent after DEB-TACE and a lower rate of liver function impairment or SAEs was observed as compared with previous studies (Malagari et al, 2008; Lammer et al, 2010), probably due to the lower doses of anticancer drugs administered. Systemic AEs, such as mucositis, alopecia, and bone marrow toxicity, were not observed in either treatment group. This safe systemic profile of cTACE could be attributed to the superselective technique we used, allowing the infusion of relatively small doses of anticancer agents to fully soak target lesion(s), and the 'on demand' treatment repetition, which reduces the likelihood of AE occurrence with respect to a pre-determined fixed schedule (Ernst et al, 1999; Jang et al, 2004).

Previous comparisons between DEB-TACE and cTACE concerning patient survival demonstrated conflicting data. The superiority of DEB-TACE is suggested by two retrospective studies (Dhanasekaran et al, 2010; Song et al, 2012). However, the first one dealt with small and differently sized groups enrolled in different periods (Dhanasekaran et al, 2010). In the second study, the 18month survival rates were $88 \%$ in DEB-TACE patients and $61 \%$ in cTACE patients, but the achievement of CR with cTACE was surprisingly rare (as already mentioned above) and the survival 


\begin{tabular}{|c|c|c|c|c|}
\hline & \multicolumn{2}{|c|}{ Univariate } & \multicolumn{2}{|c|}{ Multivariate } \\
\hline & $\mathrm{HR}(95 \% \mathrm{Cl})$ & $P$ & $\mathrm{HR}(95 \% \mathrm{Cl})$ & $P$ \\
\hline \multicolumn{5}{|l|}{ Primary therapeutic intervention } \\
\hline cTACE vs DC-Bead & $0.985(0.623-1.559)$ & 0.949 & - & - \\
\hline \multicolumn{5}{|l|}{ Clinical characteristics } \\
\hline $\begin{array}{l}\text { Age (year) } \\
\text { Male vs female } \\
\text { Viral cirrhosis vs non-viral } \\
\text { Alcohol consumption } \\
\text { Child-Pugh class B vs A class } \\
\text { ECOG } 1 \text { vs } 0 \\
\text { Serum albumin (per } \mathrm{gl}^{-1} \text { ) } \\
\text { MELD score (per unit) }\end{array}$ & $\begin{array}{l}1.024(0.992-1.057) \\
0.708(0.419-1.194) \\
1.535(0.881-2.673) \\
0.453(0.225-0.911) \\
1.889(1.053-3.387) \\
1.635(1.003-2.665) \\
0.936(0.899-0.974) \\
1.062(0.961-1.173)\end{array}$ & $\begin{array}{l}0.144 \\
0.195 \\
0.130 \\
0.026 \\
0.033 \\
0.049 \\
0.001 \\
0.239\end{array}$ & $\begin{array}{c}- \\
- \\
- \\
0.553(0.278-1.102) \\
1.109(0.560-2.196) \\
1.904(1.148-3.155) \\
0.955(0.915-0.997) \\
-\end{array}$ & $\begin{array}{c}- \\
- \\
- \\
0.092 \\
0.766 \\
0.013 \\
0.036 \\
-\end{array}$ \\
\hline \multicolumn{5}{|l|}{ Tumour burden } \\
\hline $\begin{array}{l}\text { Multiple tumours vs single tumours } \\
\text { BCLC Stage C vs B vs Stage A } \\
\text { Bilobar involvement vs monolobar } \\
\text { Largest tumour diameter (per } \mathrm{cm} \text { ) } \\
\text { Segmental injection }\end{array}$ & $\begin{array}{l}2.455(1.488-4.049) \\
1.342(1.020-1.766) \\
1.360(0.732-2.527) \\
1.002(0.989-1.014) \\
1.075(0.656-1.761)\end{array}$ & $\begin{array}{l}0.001 \\
0.035 \\
0.330 \\
0.805 \\
0.775\end{array}$ & $\begin{array}{c}2.860(1.692-4.833) \\
1.078(0.599-1.939) \\
- \\
- \\
-\end{array}$ & $\begin{array}{c}0.001 \\
0.803 \\
- \\
- \\
-\end{array}$ \\
\hline \multicolumn{5}{|c|}{$\begin{array}{l}\text { Abbreviations: } \mathrm{BCLC}=\text { Barcelona Clinic Liver Cancer; } \mathrm{Cl}=\text { confidence interval; } \mathrm{CTACE}=\text { conventional transarterial chemoembolisation; } \mathrm{DEB}=\mathrm{drug} \text {-eluting beads; } \mathrm{ECOG}=\mathrm{Eastern} \\
\text { Cooperative Oncology Group; } \mathrm{HR}=\text { hazard ratio; } \mathrm{INR}=\text { international normalized ratio; } \mathrm{MELD}=\text { model for end-stage liver disease. Variables with a } P \text {-value of }<0.10 \text { at univariate analysis } \\
\text { were entered in multivariate Cox regression. }\end{array}$} \\
\hline
\end{tabular}

analysis after the first year of follow-up was based on a very low number of cases at risk (Song et al, 2012). Conversely, a small randomised, single-centre study failed to demonstrate differences between the two techniques in terms of TTP and tumour recurrence and overall survival (Sacco et al, 2011). Finally, statistically significant better overall survival after cTACE with respect to DEB-TACE has emerged from another small retrospective study (Scartozzi et al, 2010).

The figures of 1- and 2-year overall survival (84.8\% and 56.2\%) and median survival projection (29 months) of our patients are within the wide range of values reported in the literature, depending on the heterogeneity of the patients enrolled regarding the aetiology of the liver disease, PS, Child-Pugh class, tumour burden and stage, and prevalence of cases in which previous treatment had failed to control cancer growth or HCC had recurred (Llovet et al, 2002; Lo et al, 2002; Varela et al, 2007; Doffoël et al, 2008; Dhanasekaran et al, 2010; Sacco et al, 2011; Burrel et al, 2012; Song et al, 2012; Takayasu et al, 2012).

The principal result of our study was that the type of TACE did not affect 2-year survival, which was instead adversely conditioned by PS 1, low serum albumin, and the presence of multiple tumours. Considering the strong prognostic role of PS, a more favourable PS scenario may explain the higher 2-year survival rates reported by other studies (Burrel et al, 2012; Malagari et al, 2012). The prognostic role of liver function has been already observed in patients undergoing DEB-TACE, with a 2-year survival rate of $88 \%$ in Child-Pugh class A and $75 \%$ in class B (Malagari et al, 2012). This suggests that the preservation of the extra-tumoural liver is essential for achieving good results with TACE. Therefore, between-arms equivalent liver toxicity was an important determinant of our results regarding survival. Concerning multinodularity, our study confirmed that multifocal hepatocarcinogenesis could decrease the efficacy of TACE, regardless of the technique utilised.

Eluting beads are innovative but costly medical devices, causing an overcost of at least $€ 600 /$ treatment for DEB-TACE over cTACE, offering equivalent clinical outcomes without any gain in hospital stay, making it difficult to justify their systematic employment.

Limitations of the study. The first limitation may derive from the enrolment interruption before reaching the planned sample size. However, it can be pointed out that an accepted reason for stopping a trial is its 'futility', suggested by interim analyses indicating that it is highly unlikely that the study will show any benefit from the experimental treatment (in our case DEB-TACE) as compared with the control treatment (cTACE) if the trial is continued (Casazza and Casella, 2012). Stopping trials for futility makes it possible to save time and research funds for other issues.

Another possible limitation originates from the sample size, which was calculated considering: (1) a 2-year survival rate of $40 \%$ in the cTACE group which was a value in between those $(31 \%$ and $63 \%)$ reported by the two randomised studies available at the time we planned the trial (Llovet et al, 2002; Lo et al, 2002), and (2) a $20 \%$ superiority of DEB-TACE, an optimistic view suggested by the theoretically superior advantages of the new procedure (Varela et al, 2007).

The third limitation is associated with the 'pragmatic design' of our trial, which allowed clinicians to utilise additional treatments at the time of TACE failure (or after a successful downstaging). We chose this approach not only for ethical reasons, but also to compare the two TACE techniques in a setting mimicking what actually happens in clinical practice. As a result, 26 out of 177 patients underwent complementary or sequential treatment. However, the distribution of additional treatments did not differ between the two TACE arms, and the great impact of liver transplant on survival was limited by censoring patients at the time of surgery. It is therefore likely that additional therapy had a similar, if any, weight in the two TACE arms.

Finally, our study included BCLC C patients, in whom the use of TACE may be debatable. However, this presence was due to the fact that we did not consider this stage as a contraindication to TACE, if the shift from B to C stage was only caused by PS-1 
Table 4. Abdominal pain and adverse event related to TACE procedure within 30 days after procedure

\begin{tabular}{|c|c|c|c|c|c|}
\hline & All patients $(n=177)$ & cTACE $(n=88)$ & DEB-TACE $(n=89)$ & $P$ & Effect size \\
\hline Post-procedural pain & 85 (48.0\%) & $63(71.6 \%)$ & $22(24.7 \%)$ & 0.001 & 1.124 \\
\hline Grade 1 & $47(26.6 \%)$ & 27 (30.7\%) & 20 (22.5\%) & 0.237 & 0.233 \\
\hline Grade 2 & 22 (12.4\%) & 20 (22.7\%) & $2(2.2 \%)$ & 0.001 & 1.416 \\
\hline Grade 3 & $16(9.0 \%)$ & $16(18.2 \%)$ & $0(0.0 \%)$ & 0.001 & $\mathrm{Nc}$ \\
\hline Fever & $17(9.6 \%)$ & $10(11.4 \%)$ & $7(7.9 \%)$ & 0.457 & 0.224 \\
\hline Grade 1 & $6(3.4 \%)$ & $5(5.7 \%)$ & $1(1.1 \%)$ & 0.118 & 0.933 \\
\hline Grade 2 & $6(3.4 \%)$ & $4(4.5 \%)$ & $2(2.2 \%)$ & 0.444 & 0.408 \\
\hline Grade 3 & $5(2.8 \%)$ & $1(1.1 \%)$ & $4(4.5 \%)$ & 0.243 & 0.784 \\
\hline Pain & 15 (8.5\%) & $11(12.5 \%)$ & $4(4.5 \%)$ & 0.064 & 0.612 \\
\hline Grade 1 & $5(2.8 \%)$ & $3(3.4 \%)$ & $2(2.2 \%)$ & 0.999 & 0.247 \\
\hline Grade 2 & $6(3.4 \%)$ & $5(5.7 \%)$ & $1(1.1 \%)$ & 0.118 & 0.933 \\
\hline Grade 3 & $4(2.3 \%)$ & $3(3.4 \%)$ & $1(1.1 \%)$ & 0.368 & 0.635 \\
\hline Fatigue & $4(2.3 \%)$ & $4(4.5 \%)$ & $0(0.0 \%)$ & 0.059 & Nc \\
\hline Grade 1 & $1(0.6 \%)$ & $1(1.1 \%)$ & $0(0.0 \%)$ & 0.497 & Nc \\
\hline Grade 2 & $3(1.7 \%)$ & $3(3.4 \%)$ & $0(0.0 \%)$ & 0.121 & Nc \\
\hline Nausea/vomiting & $5(2.8 \%)$ & $3(3.4 \%)$ & $2(2.2 \%)$ & 0.682 & 0.247 \\
\hline Grade 2 & $5(2.8 \%)$ & $3(3.4 \%)$ & $2(2.2 \%)$ & & \\
\hline Haematoma & $4(2.3 \%)$ & $3(3.4 \%)$ & $1(1.1 \%)$ & 0.368 & 0.635 \\
\hline Grade 1 & $4(2.3 \%)$ & $3(3.4 \%)$ & $1(1.1 \%)$ & & \\
\hline Cholecystitis & $3(1.7 \%)$ & $1(1.1 \%)$ & $2(2.2 \%)$ & 0.999 & 0.388 \\
\hline Grade 2 & $2(1.1 \%)$ & $1(1.1 \%)$ & $1(1.1 \%)$ & 0.999 & 0.000 \\
\hline Grade 3 & $1(0.6 \%)$ & $0(0.0 \%)$ & $1(1.1 \%)$ & 0.999 & $\mathrm{Nc}$ \\
\hline Necrosis (spleen infarction) & $1(0.6 \%)$ & $0(0.0 \%)$ & $1(1.1 \%)$ & 0.999 & Nc \\
\hline Grade 1 & $1(0.6 \%)$ & $0(0.0 \%)$ & $1(1.1 \%)$ & & \\
\hline Infection & $1(0.6 \%)$ & $1(1.1 \%)$ & $0(0.0 \%)$ & 0.497 & Nc \\
\hline Grade 5 & $1(0.6 \%)$ & $1(1.1 \%)$ & $0(0.0 \%)$ & & \\
\hline Allergic reaction & $2(1.1 \%)$ & $1(1.1 \%)$ & $1(1.1 \%)$ & 0.999 & \\
\hline Grade 1 & $2(1.1 \%)$ & $1(1.1 \%)$ & $1(1.1 \%)$ & & 0.000 \\
\hline Increased $\mathrm{ALT}^{\mathrm{a}}$ & $3(1.7 \%)$ & $3(3.4 \%)$ & $0(0.0 \%)$ & 0.121 & $\mathrm{Nc}$ \\
\hline Grade 1 & $2(1.1 \%)$ & $2(2.3 \%)$ & $0(0.0 \%)$ & 0.246 & Nc \\
\hline Grade 2 & $1(0.6 \%)$ & $1(1.1 \%)$ & $0(0.0 \%)$ & 0.497 & Nc \\
\hline Liver abscess & $2(1.1 \%)$ & $1(1.1 \%)$ & $1(1.1 \%)$ & 0.999 & 0.000 \\
\hline Grade 2 & $2(1.1 \%)$ & $1(1.1 \%)$ & $1(1.1 \%)$ & & \\
\hline Liver function worsening $^{\text {a }}$ & $6(3.4 \%)$ & $5(5.7 \%)$ & $1(1.1 \%)$ & 0.118 & 0.933 \\
\hline Other complications & $7(4.0 \%)$ & $4(4.5 \%)$ & $3(3.4 \%)$ & 0.720 & 0.161 \\
\hline At least one severe $A E$ & $11(6.2 \%)$ & $5(5.7 \%)$ & $6(6.7 \%)$ & 0.999 & 0.095 \\
\hline
\end{tabular}

which, in the ECOG definition, indicated patients 'unable to carry out a strenuous exercise but able to perform the usual activities of normal life'. As a matter of fact, many centres do not consider PS 1 an excluding factor for intra-arterial treatments (Lammer et al, 2010; Malagari et al, 2012), and such a position has been recently endorsed by the Italian guidelines for HCC managements (Bolondi et al, 2013). Nevertheless, this point can be considered in comparing our results with those of other studies using the BCLC B stage and PS1 as thresholds for TACE treatment.

In conclusion, the present study failed to demonstrate a superiority of DEB-TACE over cTACE in terms of efficacy, safety and, more importantly, 2-year survival. The only benefit of DEB-TACE was a lower incidence and intensity of postprocedural abdominal pain. However, since this did not affect the length of in-hospital stay and patient acceptance of additional TACEs, this marginal advantage is offset, in our opinion, by the higher cost of the new technique. Hence, the routine use of DEB-TACE in clinical practice is debatable, unless further studies can identify patient subgroups in which its use is more beneficial for outcome.

OTHER MEMBERS OF THE PRECISION ITALIA STUDY GROUP

Department of Digestive Disease and Internal Medicine, S. Orsola-Malpighi Hospital, University of Bologna: Cristina Mosconi, Alberta Cappelli, Maria Cristina Galaverni (Radiology 
Unit); Patrizia Pini, Fabio Piscaglia, Francesca Benevento, Luca Giampaolo, Claudia Caprara, Rita Menichella, Marco Lenzi (Internal Medicine Unit); Mauro Bernardi, Valentina Santi, Virginia Erroi (Medical Semeiotics Unit); Giuseppe Mazzella, Antonio Colecchia, Lucia Montrone, Ramona Schiumerini (Gastroenterology Unit); Giampaolo Bianchi, Marco Zoli (Internal Medicine Unit); Gabriella Martini, Lucilla Fraticelli, Claudia Sama, Sonia Berardi, Carla Serra (Unit of Clinical Assistance to Liver Transplant patients).

Diagnostic and Interventional Radiology-Department of Oncology Transplants and Advanced Technologies in Medicine, University of Pisa: Claudio Vignali, Francesca Bianchi, Valentina Vallini (Radiology Unit), Paola Carrai, Franco Filipponi (Hepatic surgery and liver transplantation), Sara Moneta (Infectious diseases - Campo di Marte Hospital, Pisa), Enrica Pea (Internal Medicine), Stefano Giunta (Gastroenterology - Versilia hospital - Lucca), Rodolfo Sacco, Angelo Ricchiuti, Giorgia di Fluri, (Gastroenterology and Metabolic Diseases - Pisa University Hospital) Barbara Coco (Hepatology, Pisa University Hospital).

Diagnostic Imaging Division-Department of Medical and Surgical Disciplines, AOU S. Giovanni Battista, University of Turin: Denis Rossato, Cristina Vighetti, Giacomo Battisti, Alessandro Rapellino (Radiology Unit); Patrizia Carucci, Loretta Cosso, Mario Rizzetto (Gastroenterology and Hepatology); Stefano Mirabella, Luisa Sturniolo, Giorgia Catalano, Mauro Salizzoni (Liver Transplant Center, General Surgery).

Ospedali Riuniti di Bergamo: Roberto Agazzi, Simone Limonta (Radiology Unit), Stefano Fagiuoli, Giuliana Verga, Giulia Magini (Gastroenterology Unit).

S. Maria della Misericordia Hospital, Udine: Giuseppe Aprile (Oncology) Alessandro Vit (Diagnostic and Interventional Radiology Unit).

\section{CONFLICT OF INTEREST}

The authors declare no conflict of interest.

\section{REFERENCES}

Bolondi L, Cillo U, Colombo M, Craxì A, Farinati F, Giannini EG, Golfieri R, Levrero M, Pinna AD, Piscaglia F, Raimondo G, Trevisani F, Bruno R, Caraceni P, Ciancio A, Coco B, Fraquelli M, Rendina M, Squadrito G, Toniutto P (2013) Position paper of the Italian Association for the Study of the Liver (AISF): The multidisciplinary clinical approach to hepatocellular carcinoma. Dig Liver Dis 45: 712-723.

Bruix J, Llovet JM (2002) Prognostic prediction and treatment strategy in hepatocellular carcinoma. Hepatology 35: 519-524.

Bruix J, Sherman M (2005) Practice Guidelines Committee, American Association for the Study of Liver Diseases. Management of hepatocellular carcinoma. Hepatology 42: 1208-1236.

Burnand B, Kernan WN, Feinstein AR (1990) Indexes and boundaries for "quantitative significance" in statistical decisions. J Clin Epidemiol 43: 1273-1284.

Burrel M, Reig M, Forner A, Barrufet M, de Lope CR, Tremosini S, Ayuso C, Llovet JM, Real MI, Bruix J (2012) Survival of patients with hepatocellular carcinoma treated by transarterial chemoembolisation (TACE) using Drug Eluting Beads. Implications for clinical practice and trial design. J Hepatol 56: 1330-1335.

Casazza G, Casella F (2012) Can we trust in trials stopped early for benefit? Intern Emerg Med 7: 559-561.

Dhanasekaran R, Kooby DA, Staley CA, Kauh JS, Khanna V, Kim HS (2010) Comparison of conventional transarterial chemoembolization (TACE) and chemoembolization with doxorubicin drug eluting beads (DEB) for unresectable hepatocelluar carcinoma (HCC). J Surg Oncol 101: 476-480.

Doffoël M, Bonnetain F, Bouché O, Vetter D, Abergel A, Fratté S, Grangé JD, Stremsdoerfer N, Blanchi A, Bronowicki JP, Caroli-Bosc FX, Causse X, Masskouri F, Rougier P, Bedenne L (2008) Multicentre randomised phase
III trial comparing Tamoxifen alone or with Transarterial Lipiodol Chemoembolisation for unresectable hepatocellular carcinoma in cirrhotic patients (Fédération Francophone de Cancérologie Digestive 9402). Eur J Cancer 44: 528-538.

El-Serag HB (2012) Epidemiology of viral hepatitis and hepatocellular carcinoma. Gastroenterology 142: 1264-1273.

Ernst O, Sergent G, Mizrahi D, Delemazure O, Paris JC, L’Herminé C (1999) Treatment of hepatocellular carcinoma by transcatheter arterial chemoembolization: comparison of planned periodic chemoembolization and chemoembolization based on tumor response. Am J Roentgenol 172: 59-64.

Fleiss JL (1973) Statistical Methods for Rates and Proportions. John Wiley \& Sons: New York.

Gao S, Yang Z, Zheng Z, Yao J, Deng M, Xie H, Zheng S, Zhou L (2013) Doxorubicin-eluting bead versus conventional TACE for unresectable hepatocellular carcinoma: a meta-analysis. Hepatogastroenterology 60: 813-820.

Golfieri R, Renzulli M, Mosconi C, Forlani L, Giampalma E, Piscaglia F, Trevisani F, Bolondi L. Bologna Liver Oncology Group (BLOG) (2013) Hepatocellular carcinoma responding to superselective transarterial chemoembolization: an issue of nodule dimension? J Vasc Interv Radiol 24: $509-517$.

Jang MK, Lee HC, Kim IS, Lim YS, Chung YH, Lee YS, Sung KB, Yoon HK, Ko GY, Kim AY, Suh DJ (2004) Role of additional angiography and chemoembolization in patients with hepatocellular carcinoma who achieved complete necrosis following transarterial chemoembolization. J Gastroenterol Hepatol 19: 1074-1080.

Lammer J, Malagari K, Vogl T, Pilleul F, Denys A, Watkinson A, Pitton M, Sergent G, Pfammatter T, Terraz S, Benhamou Y, Avajon Y, Gruenberger T, Pomoni M, Langenberger H, Schuchmann M, Dumortier J, Mueller C, Chevallier P, Lencioni R. PRECISION V Investigators (2010) Prospective randomized study of doxorubicineluting-bead embolization in the treatment of hepatocellular carcinoma: results of the PRECISION V study. Cardiovasc Intervent Radiol 33: 41-52.

Lencioni R, Llovet JM (2010) Modified RECIST (mRECIST) assessment for hepatocellular carcinoma. Semin Liver Dis 30: 52-60.

Llovet JM, Real MI, Montaña X, Planas R, Coll S, Aponte J, Ayuso C, Sala M, Muchart J, Solà R, Rodés J, Bruix J. Barcelona Liver Cancer Group (2002) Arterial embolisation or chemoembolisation versus symptomatic treatment in patients with unresectable hepatocellular carcinoma: a randomised controlled trial. Lancet 359: 1734-1739.

Llovet JM, Bruix J (2003) Systematic review of randomized trials for unresectable hepatocellular carcinoma: chemoembolization improves survival. Hepatology 37: 429-442.

Llovet JM, Ducreux M, Lencioni R, Di Bisceglie AM, Galle PR, Dufour JF, Greten TF, Raymond E, Roskams T, De Baere T, Ducreux M, Mazzaferro V, Bernardi M, Bruix J, Colombo M, Zhu A. European Association for the Study of the Liver; European Organisation for Research and Treatment of Cancer (2012) EASL-EORTC clinical practice guidelines: management of hepatocellular carcinoma. J Hepatol 56: 908-943.

Lo CM, Ngan H, Tso WK, Liu CL, Lam CM, Poon RT, Fan ST, Wong J (2002) Randomized controlled trial of transarterial Lipiodol chemoembolization for unresectable hepatocellular carcinoma. Hepatology 35: 1164-1171.

Malagari K, Chatzimichael K, Alexopoulou E, Kelekis A, Hall B, Dourakis S, Delis S, Gouliamos A, Kelekis D (2008) Transarterial chemoembolization of unresectable hepatocellular carcinoma with drug eluting beads: results of an open-label study of 62 patients. Cardiovasc Intervent Radiol 31: $269-280$.

Malagari K, Pomoni M, Moschouris H, Bouma E, Koskinas J, Stefaniotou A, Marinis A, Kelekis A, Alexopoulou E, Chatziioannou A, Chatzimichael K, Dourakis S, Kelekis N, Rizos S, Kelekis D (2012) Chemoembolization with doxorubicin-eluting beads for unresectable hepatocellular carcinoma: five-year survival analysis. Cardiovasc Intervent Radiol 35: 1119-1128.

Marelli L, Stigliano R, Triantos C, Senzolo M, Cholongitas E, Davies N, Tibballs J, Meyer T, Patch DW, Burroughs AK (2007) Transarterial therapy for hepatocellular carcinoma: Which technique is more effective? A systematic review of cohort and randomized studies. Cardiovasc Intervent Radiol 30: 6-25.

Pocock SJ (1992) When to stop a clinical trial. BMJ 305: 235-240.

Sacco R, Bargellini I, Bertini M, Bozzi E, Romano A, Petruzzi P, Tumino E, Ginanni B, Federici G, Cioni R, Metrangolo S, Bertoni M, Bresci G, Parisi G, Altomare E, Capria A, Bartolozzi C (2011) Conventional versus 
doxorubicin-eluting bead transarterial chemoembolization for hepatocellular carcinoma. J Vasc Interv Radiol 22: 1545-1552.

Scartozzi M, Baroni GS, Faloppi L, Paolo MD, Pierantoni C, Candelari R, Berardi R, Antognoli S, Mincarelli C, Risaliti A, Marmorale C, Antico E, Benedetti A, Cascinu S (2010) Trans-arterial chemo-embolization (TACE), with either lipiodol (traditional TACE) or drug-eluting microspheres (precision TACE, pTACE) in the treatment of hepatocellular carcinoma: efficacy and safety results from a large mono-institutional analysis. J Exp Clin Cancer Res 29: 164.

Song MJ, Chun HJ, Song do S, Kim HY, Yoo SH, Park CH, Bae SH, Choi JY, Chang UI, Yang JM, Lee HG, Yoon SK (2012) Comparative study between doxorubicin-eluting beads and conventional transarterial chemoembolization for treatment of hepatocellular carcinoma. J Hepatol 57: 1244-1250.

Takayasu K, Arii S, Kudo M, Ichida T, Matsui O, Izumi N, Matsuyama Y, Sakamoto M, Nakashima O, Ku Y, Kokudo N, Makuuchi M (2012) Superselective transarterial chemoembolization for hepatocellular carcinoma. Validation of treatment algorithm proposed by Japanese guidelines. J Hepatol 56: 886-892.

Varela M, Real MI, Burrel M, Forner A, Sala M, Brunet M, Ayuso C, Castells L, Montañá X, Llovet JM, Bruix J (2007) Chemoembolization of hepatocellular carcinoma with drug eluting beads: efficacy and doxorubicin pharmacokinetics. J Hepatol 46: 474-481.

Varela M, Reig M, de la Mata M, Matilla A, Bustamante J, Pascual S, Turnes J, Aracil C, Del Val A, Pascasio JM, Rodríguez M, Bruix J (2010) Treatment approach of hepatocellular carcinoma in Spain. Analysis of 705 patients from 62 centers. Med Clin (Barc) 134: 569-576.

This work is published under the standard license to publish agreement. After 12 months the work will become freely available and the license terms will switch to a Creative Commons AttributionNonCommercial-Share Alike 3.0 Unported License. 